\title{
Emergence of traveling density waves in cyclic multiparticle transport
}

\author{
Giorgos Kanellopoulos, ${ }^{1}$ Devaraj van der Meer, ${ }^{2}$ and Ko van der Weele ${ }^{1}$ \\ ${ }^{1}$ Mathematics Department, University of Patras, 26500 Patras, Greece \\ ${ }^{2}$ Physics of Fluids Group and J.M. Burgers Center of Fluid Dynamics, Faculty of Science and Technology, University of Twente, P.O. Box 217, \\ 7500 AE Enschede, The Netherlands
}

(Received 23 January 2015; revised manuscript received 22 May 2015; published 12 August 2015)

\begin{abstract}
Multiparticle flow through a cyclic array of $K$ connected compartments with a preferential direction is found to be able to organize itself in traveling waves. This behavior is connected with the transition between uniform flow and cluster formation. When the bias in the system is large, the particles flow freely in the preferred direction, with all compartments being equally filled at all times. Conversely, when the bias is small the particles cluster together in one compartment. The transition between these two regimes is found to involve an intermediate state in which the flow exhibits a density peak traveling periodically around the system. We relate the emergence of this traveling wave to a Hopf bifurcation and analytically derive the critical value of the "symmetry parameter" at which this bifurcation occurs. This critical value proves to be independent of the number of compartments, but the width of the intermediate regime (and thus the chance of observing traveling wave solutions) decreases sharply with growing $K$. The reverse transition follows a different course and takes place at a significantly lower value of the symmetry parameter; it is an abrupt transition from a clustered state to a uniform flow without an intermediate regime of stable traveling waves.
\end{abstract}

DOI: 10.1103/PhysRevE.92.022205

PACS number(s): 45.70.-n, 47.57.Gc, 02.30.Oz

\section{INTRODUCTION}

One of the characteristic properties of inelastic manyparticle flows is the tendency to form dense clusters. This may be traced back to the fact that the particles interact with each other in a dissipative way, which causes density differences in the system to be enhanced rather than to be smoothed out. One may think, for example, of cars on the highway, which as a result of the velocity-decreasing interactions between the cars tend to form traffic jams [1,2], or of cluster formation in transport devices for granular matter (such as coal or cereals) due to the nonelastic collisions between the grains [3,4]. Depending on the parameters of the system, sometimes the flow prevails, allowing the tendency to cluster to cause only minor density variations, while in other cases the clustering wins, bringing the flow to a complete halt.

The present paper is concerned with the crossover between these two regimes, when flow and clustering compete for dominance, and about the dynamical phenomena that accompany this transition. As we shall see, one of the most prominent of these phenomena is the emergence of density enhancements ("proto-clusters") that travel around the system. The statement "around the system" should be taken literally here, since we study a model with periodic boundary conditions.

The system we will work with is depicted in Fig. 1: it is a ring of $K$ connected compartments, through which material can flow from one compartment to the next. The flow from the $k$ th compartment in the clockwise direction is governed by a flux function $F_{R}\left(n_{k}\right)$ (where $n_{k}$ stands for the normalized number density in the compartment). Likewise, the flow in the counterclockwise direction is governed by a flux function $F_{L}\left(n_{k}\right)$. The relative strength of these flux functions is expressed by the symmetry parameter $\delta$, which is 1 in the fully symmetric case when $F_{R}\left(n_{k}\right)=F_{L}\left(n_{k}\right)$ and tends to zero in the asymmetric limit $F_{R}\left(n_{k}\right) \gg F_{L}\left(n_{k}\right)$. In our system, the mobility of the particles is such that they form clusters in the case $\delta=1$, whereas they flow freely when $\delta \rightarrow 0$. At some point between these two extreme cases we find the crossover mentioned above.

The form of the flux function we work with is inspired by the specific case of agitated granular matter, but similar flux functions also hold for other multiparticle systems, such as for traffic flow where the flux function is known as the "fundamental diagram" [2]. A particularly clear example of the emergence of traveling density waves in traffic flow was given by Sugiyama et al. [5]: these authors experimentally studied the dynamics of 22 cars on a circular road with a circumference of $230 \mathrm{~m}$ (meaning that the average vehicle density exceeded the critical value above which jams form spontaneously): starting from an initial state in which the cars were homogeneously distributed over the road, all with equal speed, tiny (unavoidable) fluctuations from this state were observed to grow into marked density enhancements traveling around the system, at a well-defined velocity, in the direction opposite to that of the individual cars. These traveling density enhancements represent the early stages of a traffic jam, or in our nomenclature protoclusters. Analytical models of ring road dynamics show the same type of density waves [6-9].

Other closely related systems include pedestrian flows in ring corridors [10], the spontaneous emergence of traveling waves along a cyclic array of asymmetrically coupled nonlinear oscillators [11], or concentration oscillations observed in chemical reaction cascades with a feedback loop [12]. One paradigmatic example of the latter is the so-called Brusselator, in which two compounds with concentrations $\mathrm{X}$ and $\mathrm{Y}$ influence each other via a cyclic series of chemical reactions in which also other elements are involved [13]. At low concentrations of the auxiliary elements, $\mathrm{X}$ and $\mathrm{Y}$ settle in a stable equilibrium state, but when the concentration of the auxiliary elements is raised above a critical level, this equilibrium state undergoes a Hopf bifurcation and the concentrations $\mathrm{X}$ and $\mathrm{Y}$ start to perform self-sustained oscillations. During the past three decades, many systems with a similar oscillatory behavior have been discovered. Today 


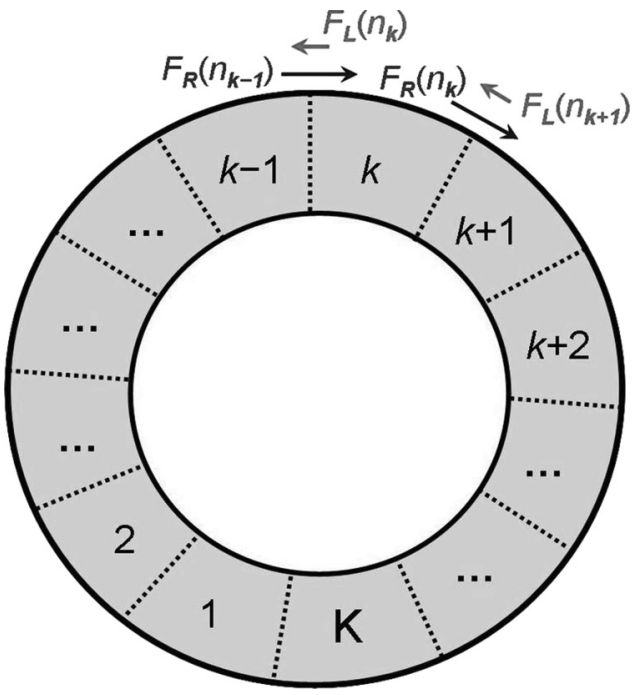

FIG. 1. Schematic top view of the cyclic system consisting of $K$ compartments. The particle flux in the clockwise direction (from compartment $k$ to $k+1$ ) is governed by the flux function $F_{R}\left(n_{k}\right)$, and in the opposite direction (from $k$ to $k-1$ ) by a smaller flux function $F_{L}\left(n_{k}\right)$; see Eq. (3). The total number of particles in the system is conserved.

more than 100 "chemical clock reactions" are known that exhibit self-sustained oscillations [12].

Also hormonal cycles (such as the testosterone production sequence controlled by the hypothalamus) operate with a built-in feedback mechanism to regulate the level of the hormone in question, and this gives rise to a nonconstant, pulsating hormone release [14]. In the same context we mention autoimmune diseases (such as rheumatoid arthritis and type I diabetes) in which the immune cells cause damage to the host tissue, inducing the release of self-antigens that further activate the immune response [12,15]. In all of these systems, the presence of a feedback loop is essential for the occurrence of a Hopf bifurcation and the associated appearance of self-sustained oscillations with a specific periodicity. The same is true for the model we study in the present paper, and vice versa: no Hopf bifurcation occurs in the absence of feedback.

The paper is organized as follows: in Sec. II we introduce the mathematical model, including the flux functions and the symmetry parameter $\delta$. In Sec. III we then study, for the relatively simple case of $K=3$ compartments, the crossover between the smooth flow regime at $\delta \ll 1$ and the clustering at $\delta=1$. It turns out that, at a certain critical value $\delta=\delta_{\mathrm{cr}}$, the uniform density profile associated with the smooth flow $\left\{n_{1}, n_{2}, n_{3}\right\}=\left\{\frac{1}{3} n_{\text {tot }}, \frac{1}{3} n_{\text {tot }}, \frac{1}{3} n_{\text {tot }}\right\}$ becomes unstable via a Hopf bifurcation, giving rise to a stable limit cycle that represents the density wave traveling around the system. This limit cycle is destabilized at some higher value of $\delta$, when its size exceeds a critical level beyond which clustering becomes inevitable. The material then heaps up in one of the three compartments [16] while the other two compartments are being emptied. In Sec. III we also explain why the transition in the reverse direction, from the clustered state to the uniform flow, is an abrupt one (without any intermediate regime of stable traveling waves) and why it takes place at a different value of $\delta$.

In Secs. IV and V we show that the same scenario also holds for $K>3$ compartments. In particular, we demonstrate that the Hopf bifurcation of the uniform flow always takes place at the same value $\delta_{\mathrm{cr}}$, irrespective of the number of compartments. We also discuss various special features that $d o$ depend on the value of $K$. One example is the pairwise limit cycle dynamics in the case when $K$ is even, which is not present when $K$ happens to be odd. Another example is the width of the limit cycle regime, which decreases rapidly with growing $K$. This means that for large values of $K$ the intermediate regime has negligible width and the transition becomes indistinguishable from an abrupt one.

Finally, in Sec. VI we close with concluding remarks and a discussion of the case of $K=4$ compartments, which presents several special features not found for any other value of $K$.

\section{MATHEMATICAL MODEL}

The model transport system described in the Introduction, with particles going from compartment to compartment via permeable walls, is governed by the following set of balance equations $(k=1, \ldots, K)$ [17-19]:

$$
\frac{d n_{k}}{d \tau}=F_{R}\left(n_{k-1}\right)-F_{L}\left(n_{k}\right)-F_{R}\left(n_{k}\right)+F_{L}\left(n_{k+1}\right),
$$

where $n_{k}(\tau)$ is the dimensionless number density in the $k$ th compartment at the instant $\tau$. We use cyclic boundary conditions: $n_{K+1}(\tau)=n_{1}(\tau)$. The above set of equations express the mass balance for each compartment: the time rate of change of $n_{k}(\tau)$ is equal to the inflow rate into this compartment minus the outflow rate. The sum of all $K$ equations is zero: $\sum_{k=1}^{k=K} d n_{k} / d \tau=0$, in agreement with the overall conservation of mass in the system. So the total number of particles is conserved:

$$
\sum_{k=1}^{k=K} n_{k}(\tau)=n_{\mathrm{tot}}
$$

with $n_{\text {tot }}$ a positive constant. The above set of equations is fully nondimensional and properly normalized $[17,18]$.

In the present paper, the flux functions will be taken to be of the form (see Fig. 2) [18]:

$$
F_{R, L}\left(n_{k}\right)=n_{k}^{2} e^{-B_{R, L} n_{k}^{2}},
$$

where the indices $R$ and $L$ denote the flow towards right and left, respectively.

The initial values of $n_{k}(0)$ (and hence the value of $n_{\text {tot }}$ ) can in principle be chosen freely, but if one wants to see the kind of competition between flowing and clustering that we are interested in, one should choose values such that $B_{L} n_{k}^{2}(0) \approx$ 1.8 on average. For the case of $B_{L}=0.7$, which we will use in many examples, this means that the initial densities lie around $n_{k}(0) \approx \sqrt{1.8 / B_{L}}=1.6$ and the corresponding value of $n_{\text {tot }}$ is simply $1.6 K$. (Note that the above typical values are all roughly of order 1 , which is a natural consequence of the fact that we study a system that has been brought into nondimensional form). 


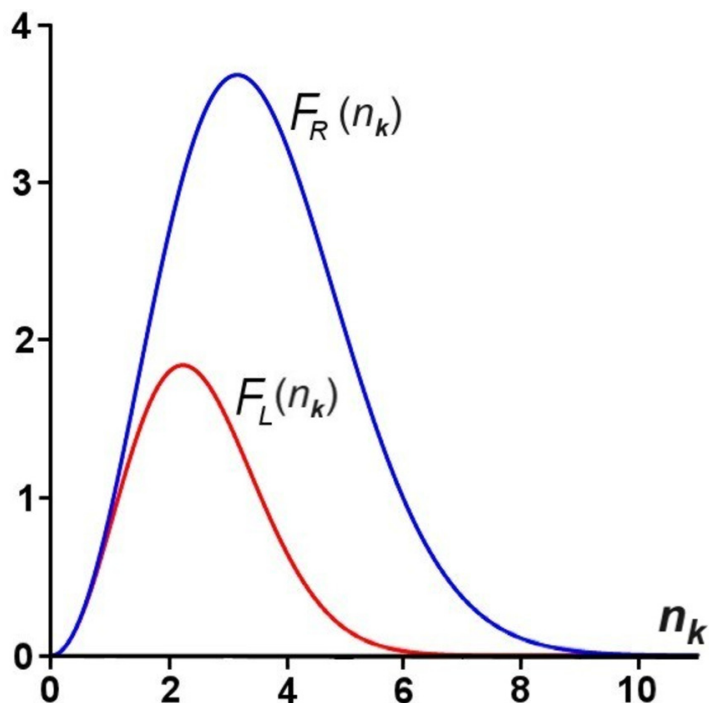

FIG. 2. (Color online) The flux functions $F_{R}\left(n_{k}\right)$ and $F_{L}\left(n_{k}\right)$ given by Eq. (3), which govern the particle flow from compartment $k$ to its neighbors at the right- and left-hand side, respectively. The parameters used for this plot are $B_{R}=0.1$ and $B_{L}=0.2$.

The specific form of the flux functions in Eq. (3) is inspired by Eggers' model for the dynamics of a granular gas in a compartmentalized setup [4], which was later generalized to describe the flow of granular matter along a conveyor belt [17-19]. The same model also represents (at least qualitatively) other multiparticle flows in which the particles interact dissipatively, such as vehicle traffic and pedestrian flows $[2,20]$. The characteristic one-humped form of the flux functions (see Fig. 2) signifies that the flow in all these systems is a nonmonotonous function of the number density $n_{k}$. It starts out from a zero flux at $n_{k}=0$ (when the compartment is simply empty) and initially grows with increasing density. However, due to the fact that the particles become less energetic when their density increases, the flux does not grow indefinitely but instead reaches a maximum (at an intermediate value of $n_{k}$ ) and thereafter goes towards zero again. It is thanks to this nonmonotonicity that clustering can occur, because this requires that the total flux from a well-filled compartment (for a large value of $n_{k}$ ) be balanced by the fluxes from the almost empty neighboring compartments (with small $n_{k}$ ).

Evidently, the parameters $B_{R}$ and $B_{L}$ play an important role here. They are a measure of the difficulty with which the particles flow (in the right and left directions, respectively), which depends on the amount of dissipation involved in each interaction and on the permeability of the walls to the right and left $[18,21]$. For instance, for $B_{R}=0$ the right wall would be fully permeable. In our system, however, both $B_{R}$ and $B_{L}$ will generally have a nonzero value.

The ratio between $B_{R}$ and $B_{L}$ indicates how symmetric the system is. Without loss of generality we will assume that $B_{R} \leqslant B_{L}$ (if there is any preferred direction at all, we take it to be the clockwise direction) and introduce the symmetry parameter $\delta$ :

$$
\delta=\frac{B_{R}}{B_{L}}, \text { with } 0 \leqslant \delta \leqslant 1
$$
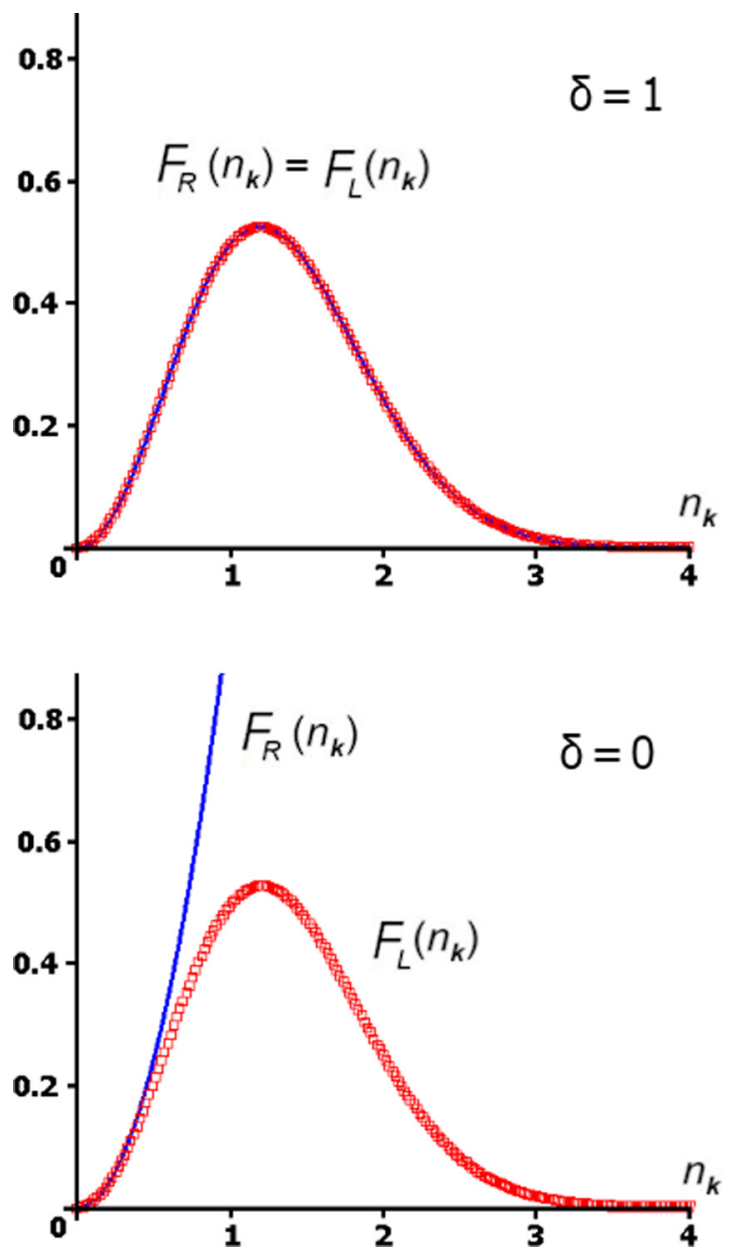

FIG. 3. (Color online) The flux functions $F_{R}\left(n_{k}\right)$ and $F_{L}\left(n_{k}\right)$ in the two limiting cases of the symmetry coefficient $\delta$ : For $\delta=1$ (symmetric case with $B_{R}=B_{L}$ ) the flux to the right is identical to the flux to the left, while for $\delta=0$ (fully asymmetric case with $B_{R}=0$ ) the material flows towards the right without any limitations. The flux towards the left is the same in both cases, with $B_{L}=0.7$.

In Fig. 3 we show the flux functions $F_{R, L}\left(n_{k}\right)$ for the two limiting cases $\delta=1$ and $\delta=0$, respectively. For $\delta=1$ the flow is fully symmetric: the flux to the left is equal to the flux to the right, $F_{R}\left(n_{k}\right)=F_{L}\left(n_{k}\right)$. In the opposite limit for $\delta=0$ the

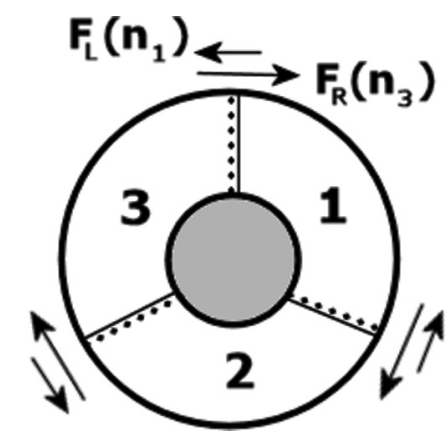

FIG. 4. Top view of the system with $K=3$ compartments studied in Sec. III. The flux in the clockwise direction is larger than in the opposite direction, as the length of the arrows indicates. 
parameter $B_{R}$ is zero. In this case, as noted above, the particles meet no difficulty at all in the clockwise direction. The flux function $F_{R}\left(n_{k}\right)$ [Eq. (3)] then loses its exponential tail and reduces to the monotonically increasing form $F_{R}\left(n_{k}\right)=A n_{k}^{2}$.

The above two limiting cases have been studied extensively: For the symmetric system $(\delta=1)$ it was found that, if the parameter $B_{R}=B_{L}$ exceeds a certain critical threshold, the particles cluster together in one compartment [22-25]. (For details and various interesting variations, for instance, in zero gravity, or using bidisperse granular mixtures, we refer to the extensive literature on the symmetric case; see Refs. [26-31] and references therein.) On the other hand, for the fully asymmetric system $(\delta=0)$ it was found that no clustering occurs and the particles collectively flow to the right [17]. In the present study we will focus on the crossover between these two limiting behaviors.

\section{THREE COMPARTMENTS}

\section{A. Phenomenology}

Given the fact that the case with $K=1$ compartment is trivial, and the case with $K=2$ compartments is by definition symmetric (in the clockwise and counterclockwise rotational sense), the smallest system in which we can study the crossover from symmetric to asymmetric behavior is that with $K=3$ compartments, see Fig. 4.

For the time being we set $B_{L}=0.7$ in order to restrict the system dependency to one parameter only ( $\delta$ or equivalently $\left.B_{R}\right)$. As initial condition we take a slightly perturbed homogeneous distribution; a good choice [see our discussion below Eq. (2)] is, e.g., $n_{1}(0)=1.6, n_{2}(0)=1.7, n_{3}(0)=1.6$.

Numerical evaluation of the equations of motion (1) shows that the transition from free-flow behavior to clustering occurs in a relatively narrow interval around $\delta=0.40$. In Fig. 5 we show the evolution of the densities $n_{1}(\tau), n_{2}(\tau)$, and $n_{3}(\tau)$ for three representative values:

(i) For $\delta=0.370$ [Fig. 5(a)] we see a rapid convergence to a uniform flow profile. Already after 60 time steps the three densities have, for all practical purposes, become equal, i.e., $n_{1}(\tau)=n_{2}(\tau)=n_{3}(\tau)=1.6333$ for all $\tau \geqslant 60$. In the inset figure we witness in the three-dimensional density space (from the initial condition at $\tau=0$ to the long-time limit for $\tau=5000$ ) how the system converges to the steady state. The observant reader may note that the dynamics in density space actually takes place on the two-dimensional surface defined by $n_{1}+n_{2}+n_{3}=n_{\text {tot }}$; we will come back to this later.

(ii) As we make our system more symmetric, when $\delta=$ 0.388 [Fig. 5(b)] we see that the excess material is passed from one compartment to the next in a periodic manner. This exchange goes on indefinitely, as can be seen also from the inset picture (for $\tau=0$ to $\tau=5000$ ), where we see that the densities now trace out a stable limit cycle.

(iii) Finally, for $\delta=0.411$ [Fig. 5(c)] we witness that the system is incapable of maintaining the limit cycle, and, after approximately 50 time steps, most of the particles cluster together inside the first compartment while the other two are nearly emptied. This is confirmed by the orbit in the density space, which evolves to a point very close to $\left\{n_{\text {tot }}, 0,0\right\}$. Note
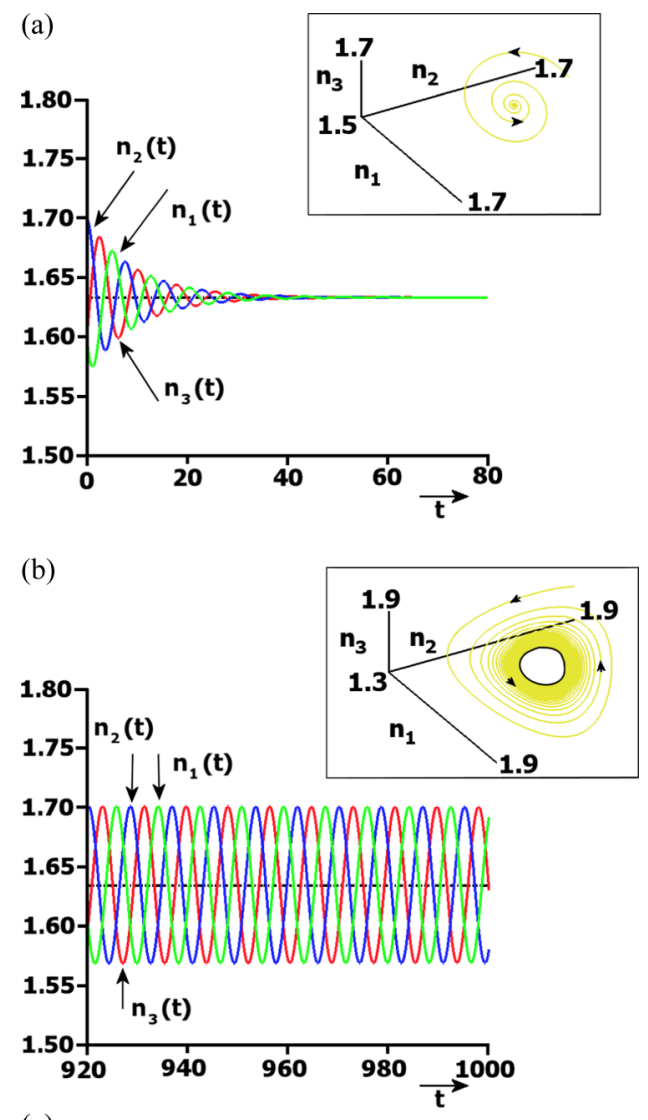

(c)

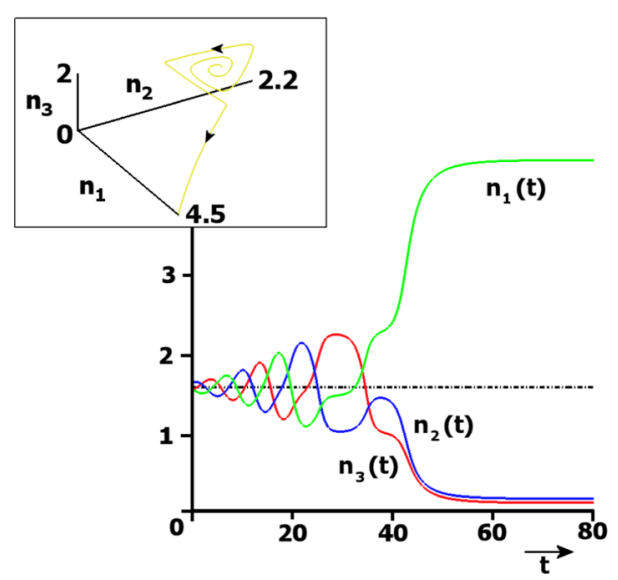

FIG. 5. (Color online) Phenomenology of the system with $K=3$ compartments at three successive values of the symmetry parameter. (a) $\delta=0.370$ : The three densities $\left\{n_{1}(t), n_{2}(t), n_{3}(t)\right\}$ are seen to converge to the evenly distributed equilibrium state indicated by the horizontal dashed line. After 60 time units they have become indistinguishable from each other. Their trajectories in the threedimensional density space spiral inward to a fixed point; see figure inset. (b) $\delta=0.388$ : The densities, instead of converging to the uniform state, now vary periodically. The density maximum is being transferred from one compartment to the next, thus forming a density wave that travels around the system. In the inset this behavior shows up as a stable limit cycle. The main plot depicts a time interval when the convergence to the limit cycle is fully completed. (c) $\delta=0.411$ : The limit cycle has become unstable, and the system is seen to form a cluster in one single compartment, while the other two are left practically empty. This is also clearly visible in the inset. Note the different scale of the vertical axis in this case. 
that, for other initial conditions, the cluster could also have formed in the second or third compartment.

In the next subsection we will explain the above observations, starting with a linear stability analysis of the uniform distribution.

\section{B. Stability analysis}

In the case of three compartments $(K=3)$, the system of coupled ODEs (1) takes the form

$$
\begin{aligned}
& \frac{d n_{1}}{d \tau}=F_{R}\left(n_{3}\right)-F_{L}\left(n_{1}\right)-F_{R}\left(n_{1}\right)+F_{L}\left(n_{2}\right), \\
& \frac{d n_{2}}{d \tau}=F_{R}\left(n_{1}\right)-F_{L}\left(n_{2}\right)-F_{R}\left(n_{2}\right)+F_{L}\left(n_{3}\right), \\
& \frac{d n_{3}}{d \tau}=F_{R}\left(n_{2}\right)-F_{L}\left(n_{3}\right)-F_{R}\left(n_{3}\right)+F_{L}\left(n_{1}\right) .
\end{aligned}
$$

An obvious fixed point of the above system, that always exists, though not necessarily stable, is the evenly distributed profile. Indeed, when all three particle densities are equal, $n_{1}=n_{2}=n_{3}=n\left(=\frac{1}{3} n_{\text {tot }}\right)$, their time derivatives $d n_{k} / d \tau(k=1,2,3)$ become identically zero. In order to study the stability of this uniform profile we consider the Jacobian matrix of the system (5) in the fixed point [noting that the flux functions defined by Eq. (3) are indeed differentiable]:

$$
\begin{aligned}
& J=\left[\begin{array}{ccc}
-\frac{d}{d n_{1}}\left[F_{R}\left(n_{1}\right)+F_{L}\left(n_{1}\right)\right] & \frac{d}{d n_{2}} F_{L}\left(n_{2}\right) & \frac{d}{d n_{3}} F_{R}\left(n_{3}\right) \\
\frac{d}{d n_{1}} F_{R}\left(n_{1}\right) & -\frac{d}{d n_{2}}\left[F_{R}\left(n_{2}\right)+F_{L}\left(n_{2}\right)\right] & \frac{d}{d n_{3}} F_{L}\left(n_{3}\right) \\
\frac{d}{d n_{1}} F_{L}\left(n_{1}\right) & \frac{d}{d n_{2}} F_{R}\left(n_{2}\right) & -\frac{d}{d n_{3}}\left[F_{R}\left(n_{3}\right)+F_{L}\left(n_{3}\right)\right]
\end{array}\right]_{\left\{n_{1}, n_{2}, n_{3}\right\}=\{n, n, n\}} \\
& =\left[\begin{array}{ccc}
-(R+L) & L & R \\
R & -(R+L) & L \\
L & R & -(R+L)
\end{array}\right] \text {, }
\end{aligned}
$$

where, for notational convenience, we denote the derivative of $F_{R}\left(n_{k}\right)$ in the fixed point $n_{k}=n$ by the letter $R$, and the analogous derivative of $F_{L}\left(n_{k}\right)$ by the letter $L$ :

$$
\begin{gathered}
\left.R \equiv \frac{d F_{R}\left(n_{k}\right)}{d n_{k}}\right|_{n_{k}=n}, \\
\left.L \equiv \frac{d F_{L}\left(n_{k}\right)}{d n_{k}}\right|_{n_{k}=n} .
\end{gathered}
$$

The above Jacobian matrix has one real $\left(\lambda_{1}\right)$ and a pair of complex conjugate $\left(\lambda_{2,3}\right)$ eigenvalues:

$$
\lambda_{1}=0, \quad \lambda_{2,3}=-\frac{3}{2}(R+L) \pm \mathrm{i} \frac{\sqrt{3}}{2}(R-L) .
$$

The eigenvalue $\lambda_{1}=0$ is associated with the conservation of mass in our system. The corresponding eigenvector in density space is $[1,1,1]$, and any motion in this direction would mean adding or subtracting equal amounts of material to (or from) the three compartments, which would violate mass conservation.

As for the other two eigenvalues $\lambda_{2,3}$, we note that their imaginary part (corresponding to periodic behavior) becomes zero when $F_{R}(n)=F_{L}(n)$ or $\delta=1$, meaning that in the fully symmetric case no Hopf bifurcation to a limit cycle can ever occur [23]. This is true not only for the case of $K=3$ compartments, but for any value of $K$, as we will demonstrate in Sec. V.
As long as the real part of $\lambda_{2,3}$ is negative, the uniform state is stable. It becomes unstable when the real part passes through zero and turns positive. Provided the imaginary part is finite this happens via a Hopf bifurcation, in which a stable limit cycle is created [32]. Setting $\operatorname{Re}\left(\lambda_{2,3}\right)=0$, the bifurcation condition thus reads

$$
R+L=0,
$$

or equivalently $\left(d / d n_{k}\right)\left[F_{R}\left(n_{k}\right)+F_{L}\left(n_{k}\right)\right]_{\left\{n_{k}=n\right\}}=0$.

With the flux functions given by Eq. (3), and writing $B_{R}=$ $\delta_{\mathrm{cr}} B_{L}$ (since we are now focusing on the critical value of $\delta$ ), the above condition becomes

$$
\begin{array}{r}
-2 A n\left[\delta_{\mathrm{cr}} B_{L} n^{2} e^{-\delta_{\mathrm{cr}} B_{L} n^{2}}-e^{-\delta_{\mathrm{cr}} B_{L} n^{2}}\right. \\
\left.+B_{L} n^{2} e^{-B_{L} n^{2}}-e^{-B_{L} n^{2}}\right]=0 .
\end{array}
$$

Solving this equation for $\delta_{\mathrm{cr}}$ we obtain the bifurcation value in analytical form:

$$
\delta_{\mathrm{cr}}\left(B_{L}, n\right)=\frac{1-W\left[\left(B_{L} n^{2}-1\right) e^{-\left(B_{L} n^{2}-1\right)}\right]}{B_{L} n^{2}},
$$

where $W(x)$ denotes the Lambert $\mathrm{W}$ function (i.e., the inverse function of $\left.f(x)=x e^{x}[18,33]\right)$. The above expression gives the critical value of $\delta$ at which the Hopf bifurcation occurs.

The solid curve in Fig. 6 shows $\delta_{\mathrm{cr}}\left(B_{L}, n\right)$ as a function of $n$, for fixed $B_{L}=0.7$. For values of $(n, \delta)$ lying under this curve, the even distribution is stable, i.e., the densities converge to 


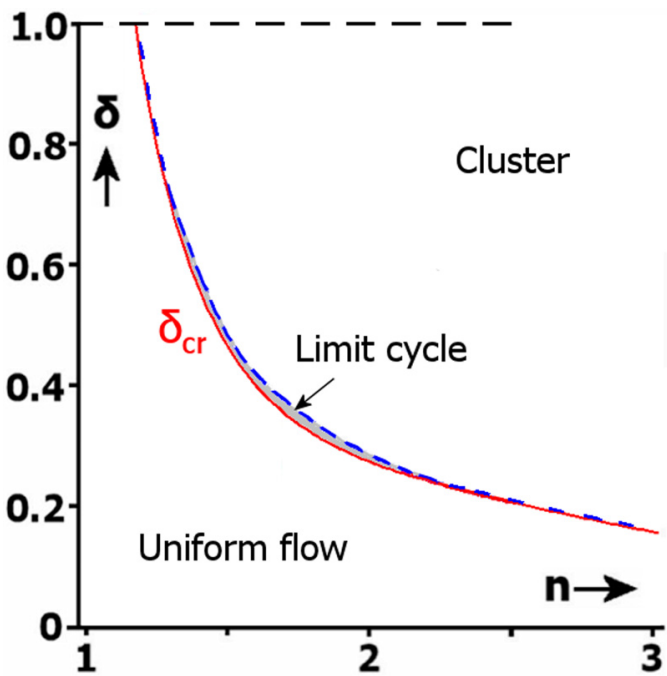

FIG. 6. (Color online) Phase diagram showing the various dynamical behaviors observed in the three-compartment system (for $\left.B_{L}=0.7\right)$ when one starts out from a flow with uniform density $n_{1}=n_{2}=n_{3}=n$. (i) For all combinations $(n, \delta)$ below the solid curve $\delta=\delta_{\text {cr }}(0.7, n)$, the uniform flow is stable; (ii) the shaded area contains the values of $(n, \delta)$ for which the uniform profile gives way to a limit cycle (i.e., a density wave traveling periodically around the system); (iii) for points $(n, \delta)$ lying above the shaded area the material forms a cluster in one compartment. The horizontal dashed line at $\delta=1$ indicates that one cannot get beyond this value.

the uniform profile as in Fig. 5(a). For $(n, \delta)$ inside the narrow shaded region, the densities converge to a stable limit cycle as in Fig. 5(b). Finally, for values of $(n, \delta)$ above the dashed line the system settles into a clustered state as in Fig. 5(c).

Figure 7 shows the corresponding bifurcation diagram for the particular value $n=1.633$ (which we also used in Fig. 5), i.e., when we follow a vertical path through Fig. 6. For $\delta<\delta_{\text {cr }}$ the uniform state is stable, indicated by the solid horizontal line, and becomes unstable via a forward (supercritical) Hopf bifurcation at $\delta=\delta_{\mathrm{cr}}=0.387498$, where the limit cycle is born. Beyond this $\delta$ value, until $\delta=0.4104$, the limit cycle increases in size, meaning that the amplitude of the traveling density wave increases. The associated wave speed decreases for increasing $\delta$, which can be understood from the fact that the tendency to flow decreases as the system becomes less biased; so the period of the limit cycle grows. As the amplitude of the density enhancement becomes larger, and the speed with which it proceeds smaller, the traveling wave increasingly looks like a "protocluster" moving slowly from one compartment to the next.

Finally, when $\delta \geqslant 0.4104$ the density inside one of the compartments will exceed the clustering threshold: the limit cycle here intersects the crossed branches corresponding to the unstable clustered state, thereby entering the basin of attraction of the stable clustered state (see also Fig. 8). In physical terms, the energy dissipation in the compartment containing the density peak becomes so large that the balance between in- and outflow can no longer be maintained. A positive net inflow into this compartment is established (at the cost of the densities of the other compartments) resulting in a cluster. The limit cycle thus loses its stability, and the system settles

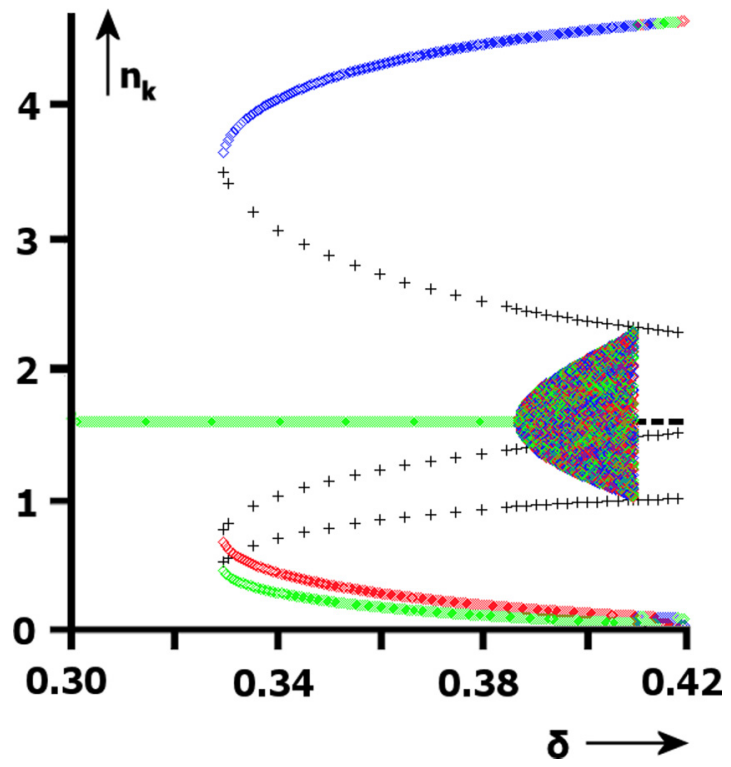

FIG. 7. (Color online) The bifurcation diagram of the threecompartment system. As the symmetry coefficient $\delta$ is increased, the uniform profile $\left\{n_{1}, n_{2}, n_{3}\right\}=(1.633,1.633,1.633)$ becomes unstable via a Hopf bifurcation at $\delta=\delta_{\mathrm{cr}}=0.387498$, where a stable limit cycle is born. Raising $\delta$ further we observe that the limit cycle increases in size, until at the value $\delta=0.4104$ it suddenly loses it stability and the system goes into the stable clustered state. This clustered state has come into existence via a saddle-node bifurcation at $\delta=0.3288$, together with its unstable counterpart, and has co-existed with the uniform state ever since. The unstable counterpart of the clustered state is responsible for the destabilization of the limit cycle; see Fig. 8.

in a stable clustered state, corresponding to the solid curved branches in the bifurcation diagram of Fig. 7.

In fact, the clustered state already existed from $\delta=0.3288$ onward, and if we would not have started out from the basin of attraction of the uniform flow (or the limit cycle) the system could have formed a cluster much earlier. Figure 7 reveals that the stable clustered state comes into existence by way of a saddle-node bifurcation (at $\delta=0.3288$ ) together with its unstable counterpart represented by the crossed curve. While the stable state develops into an increasingly pronounced cluster for growing $\delta$, the unstable clustered state becomes less pronounced. As we see in Fig. 7, when the limit cycle comes into existence (at $\delta=\delta_{\text {cr }}$ ), the density values of the unstable cluster already lie close to those of the limit cycle, and when $\delta$ is increased further they actually meet the limit cycle, rendering it unstable. This moment is illustrated in Fig. 8: the three points corresponding to (the three equivalent permutations of) the unstable clustered state coincide exactly with the corners of the triangular limit cycle. It is here that the limit cycle loses its stability and clustering sets in.

The complete sequence of events is recapitulated in the six plots of Fig. 9. As mentioned above, the dynamics in density space takes place entirely on the triangular surface defined by $n_{1}+n_{2}+n_{3}=n_{\text {tot }}$ (and $n_{1}, n_{2}, n_{3} \geqslant 0$ ), so we restrict our attention to this surface. In Fig. 9(a), at $\delta=0.320$, we see just one solid point in the center of the triangle. This corresponds to the uniform flow $n_{1}=n_{2}=n_{3}$, which at this value of 

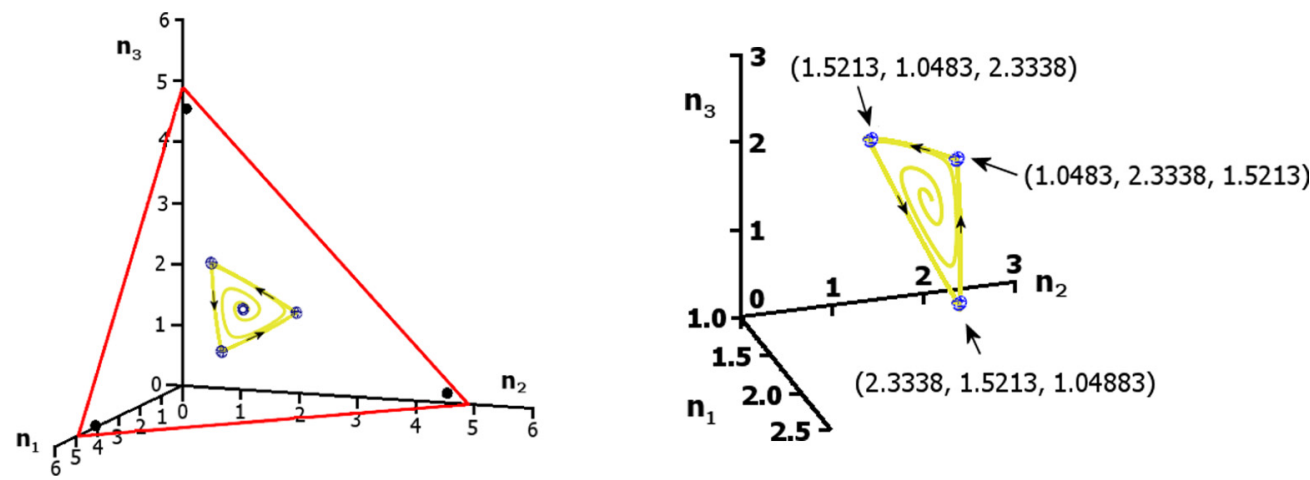

FIG. 8. (Color online) Destabilization of the limit cycle at $\delta=0.4104$. The unstable orbit which came into existence at $\delta=0.3288$ as the (unstable) counterpart of the clustered state has closed in upon the limit cycle and meets it at the point $\left\{n_{1}, n_{2}, n_{3}\right\}=(1.0483,2.3338,1.5213)$, and the two cyclic permutations of this point. At this precise moment of intersection, the limit cycle loses its stability. Note that the entire dynamics takes place on the surface defined by $n_{1}+n_{2}+n_{3}=n_{\text {tot }}=4.9$ with $n_{1}, n_{2}, n_{3} \geqslant 0$.

$\delta$ happens to be the only equilibrium state in the system. Irrespective of the initial condition, all orbits are necessarily attracted to this state: its basin of attraction is the entire surface. In Fig. 9(b), at $\delta=0.360$, six more equilibrium points have come into existence. These are the stable and unstable clustered states, denoted by solid and open dots, respectively. Evidently, the three stable clustered states are all equivalent (corresponding to the cyclic permutations of the densities over the three compartments), and the same holds for the unstable ones. The three unstable points enclose a triangular region around the fixed point in the center, which defines its new reduced basin of attraction. All initial conditions outside this triangular region lead to one of the three stable clustered states. In Fig. 9(c), at $\delta=0.389$, the central fixed point has become unstable by means of a Hopf bifurcation and it is now surrounded by a stable limit cycle. This limit cycle is traversed in the direction of the arrow, corresponding to a density elevation moving periodically from one compartment to the next. In Fig. 9(d), at $\delta=0.405$, we see how the limit cycle has expanded, and meanwhile the three unstable clustered states have moved closer to the center of the surface. In Fig. 9(e), at $\delta=0.4104$, they meet each other, and the limit cycle is destabilized (this is the same moment as depicted in Fig. 8). Finally, in Fig. 9(f) at $\delta=0.415$ we see that the only stable

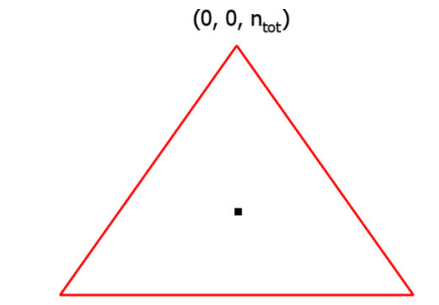

$\left(\mathrm{n}_{\text {tot }}, 0,0\right)$

(a)

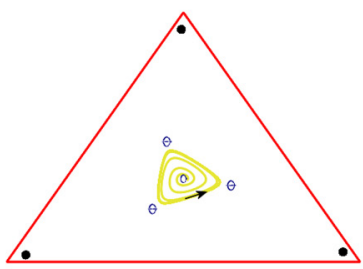

(d)

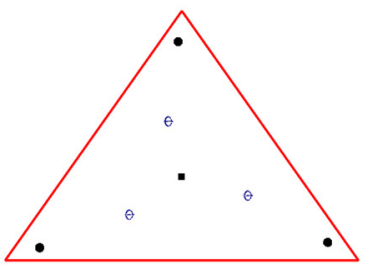

(b)

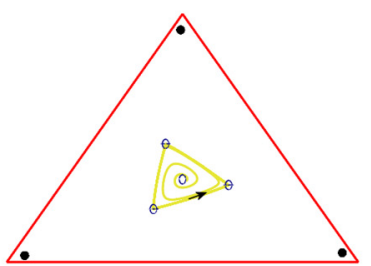

(e)

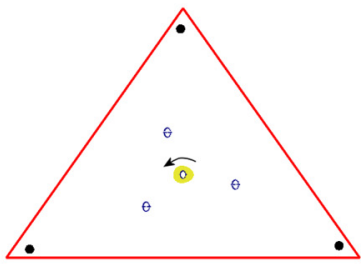

(c)

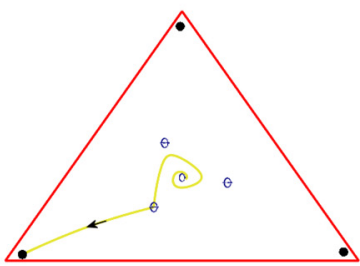

(f)

FIG. 9. (Color online) The triangular surface in density space defined by $n_{1}+n_{2}+n_{3}=n_{\text {tot }}$ (on which the dynamics of the threecompartment system takes place) for six successive values of the symmetry parameter $\delta$. (a) At $\delta=0.320$ the uniform flow (corresponding to the solid point in the center of the triangle) is the only equilibrium in the system and its basin of attraction is simply the entire triangle; (b) at $\delta=0.360$ the fixed point in the center is still stable but by now six more equilibrium points have come into existence by means of a saddle-node bifurcation, corresponding to three stable clustered states (solid dots) and their unstable counterparts (open dots); (c) at $\delta=0.389$ the fixed point has just become unstable (by means of a Hopf bifurcation), and around it we discern a stable limit cycle, which is traversed in the direction indicated by the arrow; (d) at $\delta=0.405$ we see how the three unstable clustered states and the limit cycle close in upon each other; (e) at $\delta=0.4104$ they meet each other, thereby rendering the limit cycle unstable, and finally (f) at $\delta=0.415$ we see how an orbit starting out from the neighborhood of the central fixed point (uniform flow) ends up, via one of the unstable clustered states, in the stable clustered state in the lower left corner, with almost all material in the first compartment. 


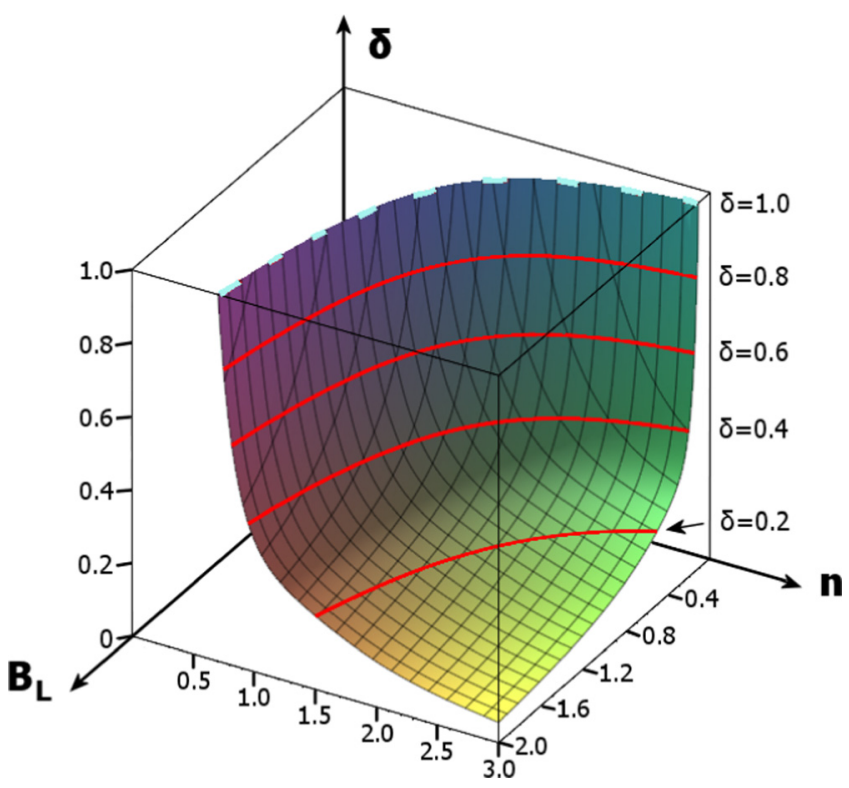

FIG. 10. (Color online) The critical surface corresponding to the Hopf bifurcation in three-dimensional $\left\{B_{L}, n, \delta\right\}$ parameter space, generated from Eq. (11). For parameter combinations below the surface the uniform flow is stable. It is seen that for sufficiently small values of $B_{L}$ and $n$ (strong shaking and small average density, respectively) the uniform flow never becomes unstable, since the surface here exceeds the upper bound $\delta=1$.

equilibria remaining in the system are the three clustered states. In the plot we see an orbit starting out from the neighborhood of the central (unstable) fixed point, which first moves to one of the unstable clustered states, and from there to the clustered state in the lower left corner. This is precisely the behavior we also witnessed in Fig. 5(c).

Let us also say a few words about the reverse transition, when $\delta$ is decreased (from 1 to 0 ), and we follow the fate of the clustered state. In this case, the system remains in the clustered state until we meet the saddle-node bifurcation at $\delta=0.3288$, where the cluster simply ceases to exist as an equilibrium state. At this point we witness a sudden collapse of the cluster [25], and the material spreads out over all compartments. This involves a transient density wave (which in the limit $\delta \rightarrow 0$ takes the form of a Burgers shock wave [17]), gradually diminishing in height until the flow becomes uniform. So we see that the crossover between the uniform flow and the clustered state is markedly hysteretic.

\section{The influence of the parameter $B_{L}$}

The results we have presented until now were all for the fixed parameter value $B_{L}=0.7$, and for a given density level $n=n_{\text {tot }} / 3=1.6333$, in order to highlight the transition from smooth flow to clustering as a function of the symmetry parameter $\delta$. In fact, however, the behavior of the system is affected also by the value of the parameter $B_{L}$ and the choice of $n=n_{\text {tot }} / 3$. In Fig. 10 we depict the critical surface corresponding to the Hopf bifurcation in three-dimensional $\left\{B_{L}, n, \delta\right\}$ parameter space, generated from Eq. (11). We see that $\delta_{\mathrm{cr}}=\delta_{\mathrm{cr}}\left(B_{L}, n\right)$ is very small when $B_{L}$ and $n$ are relatively large; this stands to reason, since large $B_{L}$ means that the system is only weakly vibrated, and large $n$ means that there are many particles in the system, both of which are favorable for clustering. Vice versa, when $B_{L}$ and $n$ are small (corresponding to strong shaking and a small number of particles, respectively) the conditions are such as to favor the uniform smooth flow; when $B_{L}$ and $n$ fall below a certain critical threshold (indicated by the dashed curve in Fig. 10) the predicted value of $\delta_{\mathrm{cr}}$ even exceeds the upper limit 1, which means that the Hopf bifurcation never occurs at all. So in this case the particles will always keep flowing, without ever forming a traveling density wave (limit cycle), let alone a cluster.

An alternative way to see this is by following the trajectories of the nonzero eigenvalues $\lambda_{2,3}$ in the complex plane. Figure 11 shows three characteristic trajectories (for three different values of $B_{L}$, keeping $n=1.633$ fixed) as the value of $\delta$ is gradually increased from 0 to 1 . In all three cases the eigenvalues are seen to start out (for $\delta=0$ ) with a negative real part, corresponding to a stable uniform flow. In the case $B_{L}=0.700$ (rightmost trajectories, relatively weak shaking) the eigenvalues cross the imaginary axis at the well-known value $\delta_{\text {cr }}=0.3875$, where the Hopf bifurcation occurs. They meet at some point along the positive real axis when $\delta=1$, long after the uniform flow they represent has become unstable.

At the value $B_{L}=0.375$ (middle trajectories, stronger shaking) the eigenvalues never really cross the imaginary axis, but just manage to reach the origin of the complex plane as $\delta \rightarrow 1$. This corresponds to the aforementioned critical threshold marked by the dashed curve in Fig. 10, at which the Hopf bifurcation ceases to exist.

For the still smaller value $B_{L}=0.275$ (leftmost trajectories, very strong shaking) the real part of the eigenvalues $\lambda_{2,3}$ remains negative right up to $\delta=1$, where they meet at some point along the negative real axis.

\section{MORE THAN THREE COMPARTMENTS}

In this section we generalize the system by increasing the number of compartments. Just as before, the total number of particles in the system is conserved. This means that, just as the dynamics for the three-compartment system took place on the two-dimensional surface defined by $\sum_{k=1}^{3} n_{k}=$ constant, for $K$ compartments the dynamics takes place on the positive segment of the $(K-1)$-dimensional hypersurface defined by $\sum_{k=1}^{K} n_{k}=$ constant.

In order to study the crossover from the free flow regime to the clustered state, we again focus on the stability of the uniform flow given by $n_{1}=n_{2}=\cdots=n_{K}=n$. Our first step is thus to determine the eigenvalues of the corresponding $K \times$ $K$ Jacobian matrix. For $K=4$ we find

$$
\begin{gathered}
\lambda_{1}=0, \quad \lambda_{2}=-2(R+L), \\
\lambda_{3,4}=-(R+L) \pm i(R-L) .
\end{gathered}
$$

Similarly for $K=5$,

$$
\begin{aligned}
\lambda_{1} & =0 \\
\lambda_{2,3} & =\frac{-5+\sqrt{5}}{4}(R+L) \pm \frac{i}{4} \sqrt{2(5+\sqrt{5})}(R-L), \\
\lambda_{4,5} & =\frac{-5-\sqrt{5}}{4}(R+L) \pm \frac{i}{4} \sqrt{2(5-\sqrt{5})}(R-L),
\end{aligned}
$$




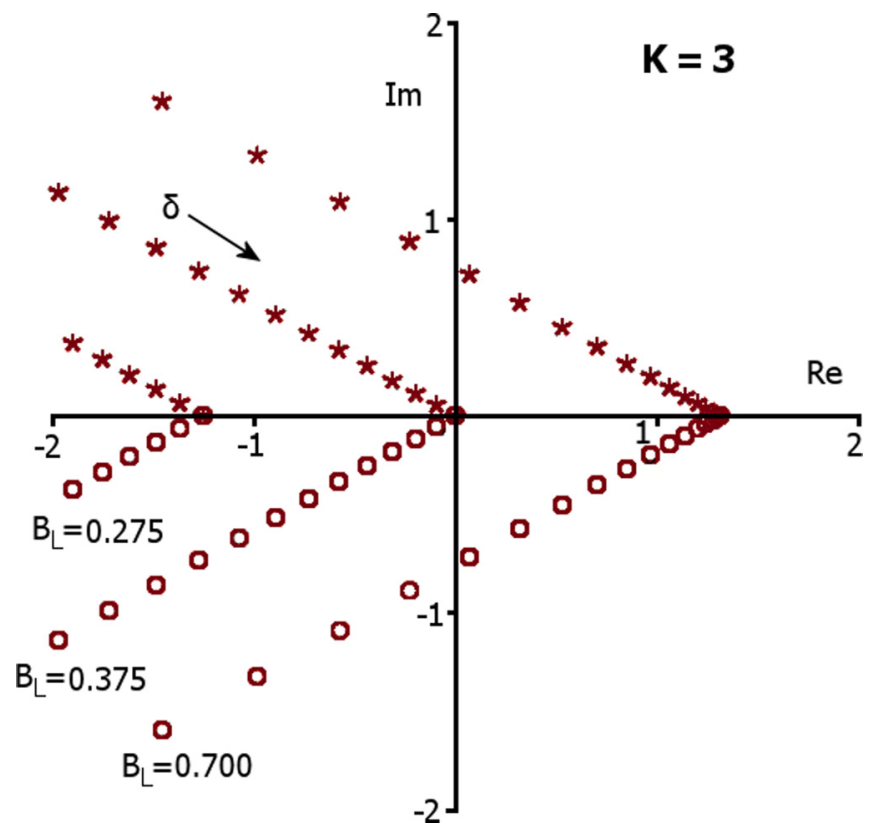

FIG. 11. (Color online) Three trajectories of the eigenvalue pair $\lambda_{2,3}$ in the complex plane, for three different values of $B_{L}$ and a fixed average density $n=1.6333$. The value of $\delta$ gradually increases from 0 to 1 along each trajectory from left to right. The rightmost trajectory corresponds to the value $B_{L}=0.700$ used in the previous figures; for this value the eigenvalue pair crosses the imaginary axis (i.e., the Hopf bifurcation occurs) at $\delta=0.3875$. The leftmost trajectory corresponds to $B_{L}=0.275$, for which the real part of the eigenvalues $\lambda_{2,3}$ remains negative right up to $\delta=1$ (where they meet at some negative value along the real axis) so no Hopf bifurcation occurs in this case. The middle trajectory corresponds to the intermediate case $B_{L}=0.375$ for which the eigenvalues meet precisely at the origin for $\delta=1$.

and for $K=6$,

$$
\begin{aligned}
\lambda_{1} & =0, \quad \lambda_{2}=-2(R+L), \\
\lambda_{3,4} & =-\frac{3}{2}(R+L) \pm i \frac{\sqrt{3}}{2}(R-L), \\
\lambda_{5,6} & =-\frac{1}{2}(R+L) \pm i \frac{\sqrt{3}}{2}(R-L) .
\end{aligned}
$$
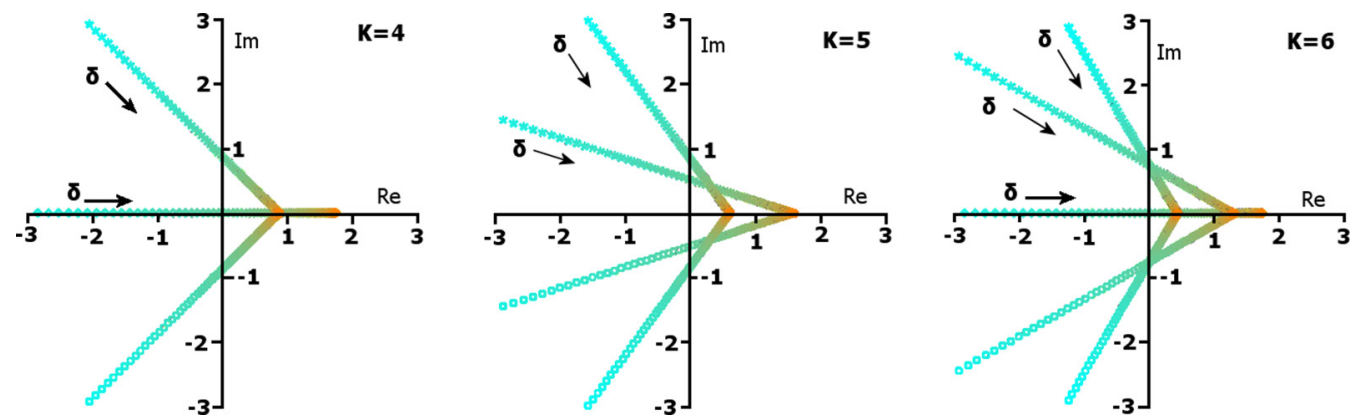

FIG. 12. (Color online) The trajectories (as function of the symmetry parameter $\delta$, which is being varied from 0 to 1 , while the other parameters are fixed at $B_{L}=0.700$ and $n=1.633$ ) for the nonzero eigenvalues of the systems with $K=4, K=5$, and $K=6$ compartments, respectively. The Hopf bifurcation (which takes place when the eigenvalues cross the imaginary axis from left to right) occurs at exactly the same value of $\delta$ in all three cases. The arrows indicate the direction of increasing $\delta$, with the color coding running from cyan (light) at $\delta=0$ to orange (dark) at $\delta=1$.

Comparing these eigenvalues with those for the case of three compartments [see Eq. (8)] we note that the real and imaginary parts are always of the same form $(R+L)$ and $(R-L)$, respectively, and only the prefactors differ. This means that the Hopf bifurcation, where the real parts become zero, occurs at the same value $\delta_{\mathrm{cr}}\left(B_{L}, n\right)$ [given by Eq. (11)] for all $K$. The eigenvalues evaluated thus far show this explicitly for $K=3, \ldots, 6$, but we can in fact prove that the same holds for an arbitrary number of compartments. The proof (including the analytical form of the eigenvalues for all possible $K$ ) will be given separately in the next section.

In Fig. 12 we show the trajectories in the complex plane of the $(K-1)$ nonzero eigenvalues for the cases $K=4,5,6$, as the symmetry parameter $\delta$ is gradually increased from 0 (left) to 1 (right). The other two system parameters $B_{L}=0.700$ and $n=1.633$ are kept fixed. We observe that all depicted eigenvalues (irrespective of the value of $K$ ) cross the imaginary axis at exactly the same value of $\delta=\delta_{\mathrm{cr}}=0.387498$. Another thing to note is the fact that for $\delta=1$, at the right-hand side of the plots, all eigenvalues fall upon the real axis. In this case, when the system is not biased towards any flow direction anymore, the system ceases to support periodic solutions (neither stable nor unstable). The vanishing of the imaginary part of the eigenvalues of the uniform flow corroborates this.

In Figs. 13 and 14 we present the bifurcation diagrams for $K=5$ and 6 compartments, along with plots of the density oscillations in the system (corresponding to the limit cycle behavior) at $\delta=\delta_{\text {cr }}+0.0001$, i.e., just after the Hopf bifurcation. The case for $K=4$ compartments will be discussed in the concluding section, since it presents some special features that make it nonrepresentative for the other values of $K$. Here we focus on the main trend.

Comparing the bifurcation diagrams for $K=5$ and 6 with the corresponding diagram for $K=3$ compartments, two major things capture the eye. First, the interval of $\delta$ for which a stable limit cycle exists is seen to decrease for growing $K$. Second, in the density oscillations for $K=6$ we see a marked tendency for pair formation (an alternating pattern of high and low densities) which is absent in the cases $K=3$ and $K=5$.

With respect to the first observation we have also investigated several cases for $K>6$ and indeed found that the interval corresponding to the stable limit cycle diminishes rapidly as $K$ grows. For instance, whereas the width of the 

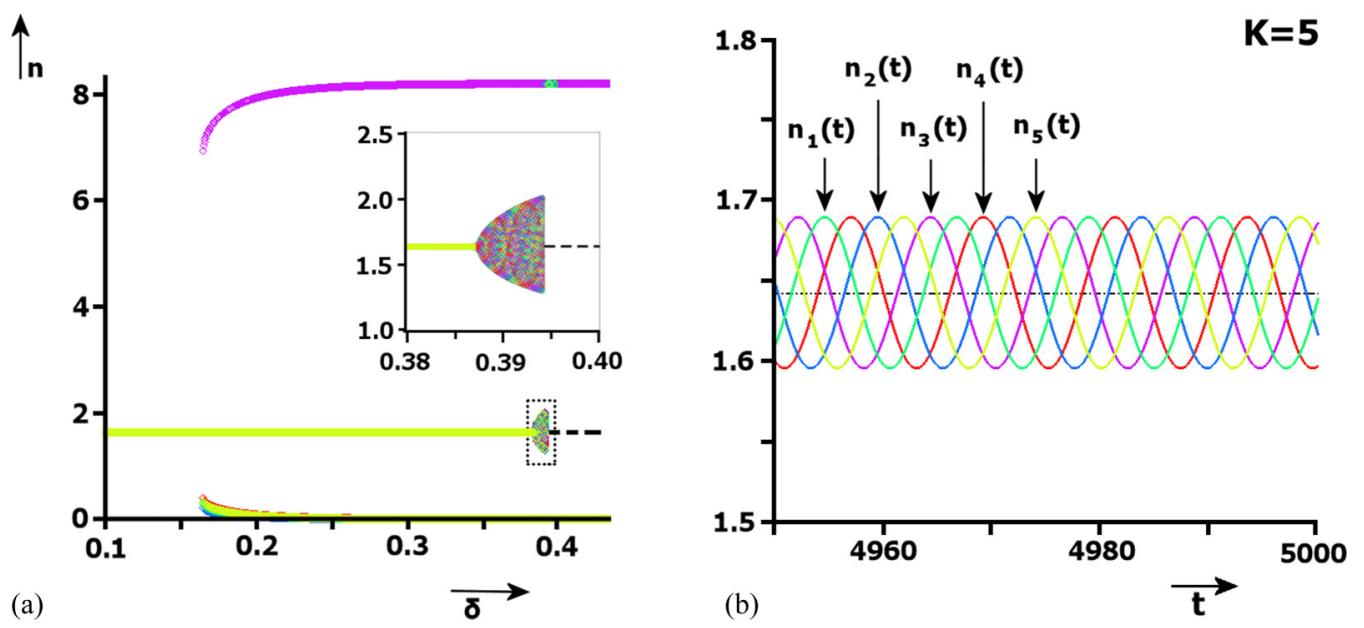

FIG. 13. (Color online) (a) Bifurcation diagram for $K=5$ compartments. For simplicity, the curves corresponding to the unstable clustered states (which are born via a saddle-node bifurcation together with the stable clustered states at $\delta=0.164$ ) have not been included. The Hopf bifurcation occurs at the same value $\delta_{\text {cr }}$ as for the three-compartment system (see Fig. 7), but the width of the interval in which the associated limit cycle is stable is significantly smaller, namely, 0.0070; see also the close-up of this region in the inset. (b) The limit cycle dynamics at $\delta=\delta_{\text {cr }}+0.0001$, just after the Hopf bifurcation. The time interval has been chosen on purpose at a well-advanced stage; this not only guarantees that the oscillatory motion has converged to its final frequency and amplitude but also serves to illustrate that the periodic exchange between the compartments goes on indefinitely.

interval for $K=3$ was found to be $\Delta \delta=0.0229$, and for $K=6$ still $\Delta \delta=0.0036$, for $K=15$ compartments it has reduced to $\Delta \delta=0.0006$, and for even longer arrays it becomes practically negligible.

This means that the transition from smooth flow to clustering for growing $K$ becomes increasingly abrupt. For large $K$ it will be quite difficult to observe the intermediate stage with the limit cycle, since it requires an extreme fine-tuning of the parameters $\delta, B_{L}$, and $n$ in a tiny zone above the surface $\delta_{\mathrm{cr}}$. So, in retrospect, we see that the case $K=3$ is not only the most convenient one for studying the transition because of its relative simplicity, but it also happens to be the case in which the phenomenon plays the biggest role.

With respect to the second observation, the alternating pattern is found not only for $K=6$ but for any even number of compartments. In fact, it represents the natural way in which granular material tends to organize itself, due to its tendency to enhance density differences; the effect is usually so pronounced that the density oscillations of the high-level compartments do not cross those of their low-level counterparts, leaving a blank zone of densities in between that are not visited; see Fig. 14. The same tendency exists when
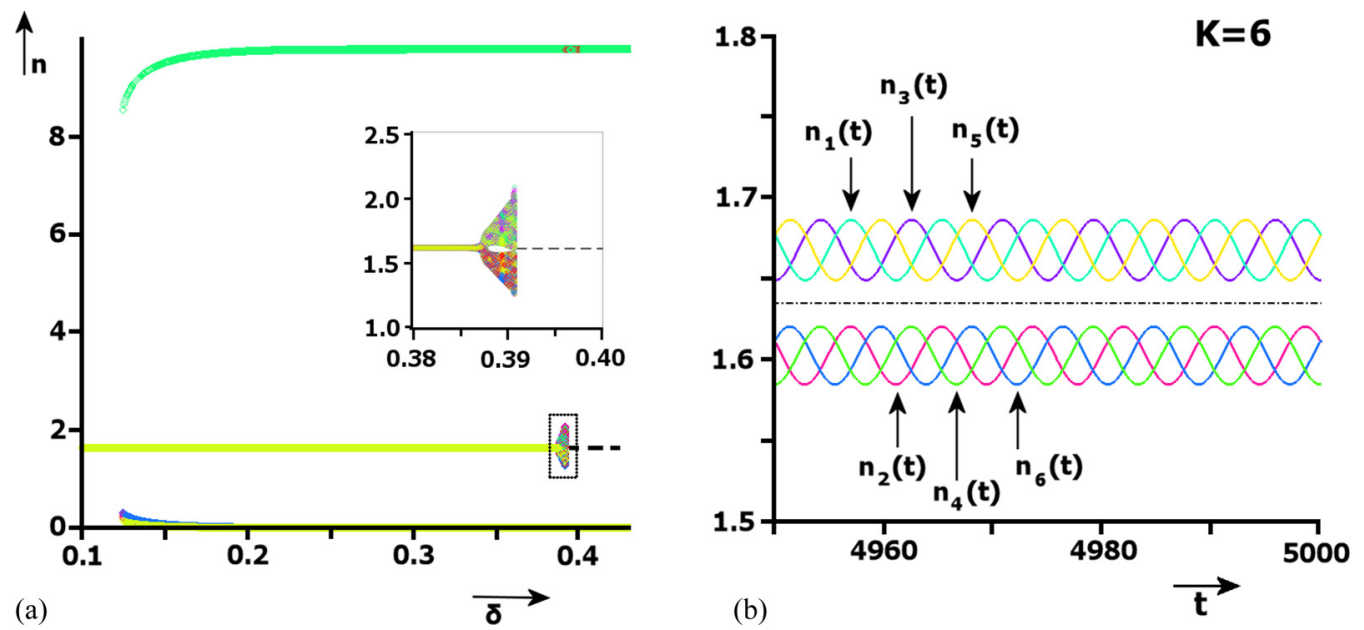

FIG. 14. (Color online) (a) Bifurcation diagram for $K=6$ compartments. In comparison with the previous value of $K$, the saddle-node bifurcation in which the clustered states are being born (together with their unstable counterparts, not depicted) has shifted still further to the left: it now takes place at $\delta=0.125$. The interval of $\delta$ associated with a stable limit cycle has again become smaller, 0.0036 ; see also the inset. In this close-up one discerns a blank zone in the region of the limit cycle, which is typical of all systems with an even number of compartments. (b) Limit-cycle dynamics at $\delta=\delta_{\text {cr }}+0.0001$, just after the Hopf bifurcation. We see that the number densities in the compartments 1,3 , and 5 are consistently larger than those in the other compartments; the intermediate density values that are not visited correspond to the blank zone observed in the inset of the bifurcation diagram of figure (a). 
the array has an odd number of compartments, but in this case the pair formation is frustrated (the odd compartment cannot simultaneously have a high and a low density), and hence the alternating pattern fails to emerge. This explains the absence of such a pattern in the long-time behavior for $K=3,5$, and all other odd values of $K$.

\section{INVARIANCE OF THE HOPF BIFURCATION WITH RESPECT TO $K$}

In this section we will prove that the Hopf bifurcation occurs at the same level of asymmetry $\left(\delta_{\mathrm{cr}}\right)$ for any number of compartments $K$. An extra result that emerges from the analysis is that the symmetric system $\left(F_{R}=F_{L}\right)$ does not support periodic density oscillations for any $K$.

Consider a system with $K \geqslant 2$ compartments; then the Jacobian $K \times K$ matrix calculated in the fixed point $\{n, \ldots, n\}$ has the following form (where, as before, $R=d F_{R} / d n$ and $L=d F_{L} / d n$ ):

$$
J_{K}=\left[\begin{array}{cccccc}
-R-L & L & 0 & 0 & \ldots & R \\
R & -R-L & L & 0 & \ldots & 0 \\
0 & R & -R-L & L & \ldots & 0 \\
\ldots & \ldots & \ldots & \ldots & \ldots & \ldots \\
0 & \ldots & 0 & R & -R-L & L \\
L & \ldots & 0 & 0 & R & -R-L
\end{array}\right] .
$$

We will show that the $K$ eigenvalues $\lambda_{j, J_{K}}(j=1, \ldots, K)$ are of the form

$$
\lambda_{j, J_{K}}=c_{j}(R+L)+d_{j}(R-L),
$$

where the coefficients $c_{j}$ and $d_{j}$ (of the real and imaginary parts, respectively) are given by

$$
\begin{aligned}
& c_{j}=-2 \sin ^{2}\left(\frac{j \pi}{K}\right), \quad j=1, \ldots, K, \\
& d_{j}=-i \sin \left(\frac{2 j \pi}{K}\right), \quad j=1, \ldots, K .
\end{aligned}
$$

It is easily checked that the sets of eigenvalues for the special cases $K=3, \ldots, 6$ presented earlier coincide with the eigenvalues given by the above general expression, only with a somewhat different ordering.

With respect to the coefficients $c_{j}$, we see that one of them $\left(c_{K}\right)$ is zero and all the others are negative real numbers. This means that, when $(R+L)$ goes through zero at $\delta=\delta_{\mathrm{cr}}$, the real parts of all nonzero eigenvalues switch simultaneously from negative to positive. This is precisely what defines the Hopf bifurcation.

With regard to the coefficients $d_{j}$, one or two of them are zero (for odd $K$ only $d_{K}$ is zero, while for even $K$ both $d_{K / 2}$ and $d_{K}$ are zero) and the rest of the $d_{j}$ are evenly divided between positive and negative imaginary values, in such a way that the corresponding complex eigenvalues come in conjugate pairs. In the present context the coefficients $d_{j}$ play a less important role than the $c_{j}$, since the $d_{j}$ (belonging to the imaginary part of the eigenvalues) are not connected to the stability of the fixed point.

Proof. Since $J_{K}$ is a real square matrix $K \times K$, it may be decomposed as follows:

$$
J_{K}=\frac{1}{2}\left(J_{K}+J_{K}^{T}\right)+\frac{1}{2}\left(J_{K}-J_{K}^{T}\right),
$$

where $J_{K}^{T}$ denotes the transpose of $J_{K}$. The term $\frac{1}{2}\left(J_{K}+J_{K}^{T}\right)$ is a real symmetric matrix with real eigenvalues (including zero), while $\frac{1}{2}\left(J_{K}-J_{K}^{T}\right)$ is a skew-symmetric matrix with purely imaginary eigenvalues (and possibly zero). Moreover,
$J_{K}$ is a normal matrix (it commutes with its transpose), and thus the matrices $\frac{1}{2}\left(J_{K}+J_{K}^{T}\right)$ and $\frac{1}{2}\left(J_{K}-J_{K}^{T}\right)$ also commute. Now, using a theorem by Fröbenius concerning commuting matrices (proved in 1878; see Ref. [34]), the eigenvalues of $J_{K}$ may be ordered in such a way as to follow the same decomposition:

$$
\lambda_{j, J_{K}}=\lambda_{j, \frac{1}{2}\left(J_{K}+J_{K}^{T}\right)}+\lambda_{j, \frac{1}{2}\left(J_{K}-J_{K}^{T}\right)}
$$

for $j=1, \ldots, K$. From Eq. (20), combined with the fact that $\frac{1}{2}\left(J_{K}+J_{K}^{T}\right)$ has only real (or zero) eigenvalues and $\frac{1}{2}\left(J_{K}-\right.$ $J_{K}^{T}$ ) has only imaginary (or zero) ones, we conclude that

$$
\operatorname{Re}\left(\lambda_{j, J_{K}}\right)=\lambda_{j, \frac{1}{2}\left(J_{K}+J_{K}^{T}\right)}, \quad j=1, \ldots, K,
$$

and

$$
\operatorname{Im}\left(\lambda_{j, J_{K}}\right)=\lambda_{j, \frac{1}{2}\left(J_{K}-J_{K}^{T}\right)}, \quad j=1, \ldots, K
$$

Now we observe that in our case $\frac{1}{2}\left(J_{K}+J_{K}^{T}\right)=(R+L) A$, with $A$ the real and symmetric $K \times K$ matrix given by

$$
A=\frac{1}{2}\left[\begin{array}{cccccc}
-2 & 1 & 0 & 0 & \ldots & 1 \\
1 & -2 & 1 & 0 & \ldots & 0 \\
0 & 1 & -2 & 1 & \ldots & 0 \\
\ldots & \ldots & \ldots & \ldots & \ldots & \ldots \\
0 & \ldots & 0 & 1 & -2 & 1 \\
1 & \ldots & 0 & 0 & 1 & -2
\end{array}\right]
$$

Therefore, if we denote the eigenvalues of $A$ by $c_{j}$, expression (21) takes the following form:

$$
\operatorname{Re}\left(\lambda_{j, J_{K}}\right)=c_{j}(R+L), \quad j=1, \ldots, K,
$$

and we note that, because $A$ is a negative semidefinite matrix for all $K$, the coefficients $c_{j}$ can never become positive [24]. The explicit form of the $c_{j}$ given in Eq. (17) confirms this.

This completes the proof of expression (16) as far as the real part is concerned.

Likewise, for the imaginary part we consider the matrix $\frac{1}{2}\left(J_{K}-J_{K}^{T}\right)=(R-L) B$, where $B$ is the skew-symmetric 

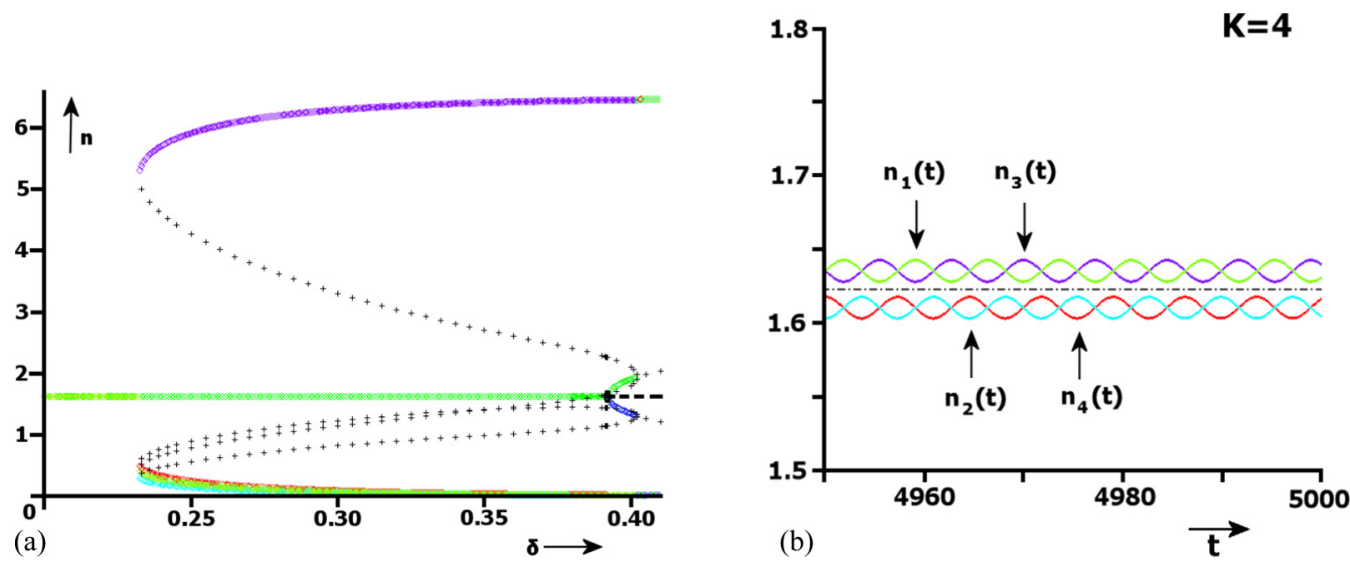

FIG. 15. (Color online) (a) Bifurcation diagram for the case of $K=4$ compartments. Unlike any other case, we see that the Hopf bifurcation is almost instantaneously followed by a transformation of the limit cycle to a stable period- 2 orbit. This orbit is rendered unstable at $\delta=0.402$ (via a reverse period doubling bifurcation) when it meets the unstable clustered state. (b) The limit cycle oscillation immediately after the Hopf bifurcation at $\delta=\delta_{\mathrm{cr}}$. At a slightly higher value of $\delta$ the amplitude of the oscillations will be reduced to zero, creating the aforementioned period-2 orbit.

$K \times K$ matrix given by

$$
B=\frac{1}{2}\left[\begin{array}{cccccc}
0 & -1 & 0 & 0 & \ldots & 1 \\
1 & 0 & -1 & 0 & \ldots & 0 \\
0 & 1 & 0 & -1 & \ldots & 0 \\
\ldots & \ldots & \ldots & \ldots & \ldots & \ldots \\
0 & \ldots & 0 & 1 & 0 & -1 \\
-1 & \ldots & 0 & 0 & 1 & 0
\end{array}\right] .
$$

Denoting the eigenvalues of $B$ by $d_{j}$, expression (22) then takes the form

$$
\operatorname{Im}\left(\lambda_{j, J_{K}}\right)=d_{j}(R-L), \quad j=1, \ldots, K
$$

where $d_{j}$ is either zero or a purely imaginary number, as is indeed verified by the explicit form (18).

This completes the proof of expression (16) also with respect to the imaginary part.

The above proof shows that the real parts of the eigenvalues $\lambda_{j}$ simultaneously switch from negative to positive, at the same value of $\delta_{\text {cr }}$ for all $K \geqslant 3$. In other words, we can use the bifurcation criterion (9) regardless of the number of compartments. Of course, it depends on the form of the flux function whether this criterion can be solved analytically. For the Eggers-type flux functions used in the present paper this can be done [see Eq. (11)], whereas for other choices one generally will have to resort to numerical solutions.

As far as the imaginary part of the eigenvalues is concerned, the form of Eq. (26) confirms our earlier observation that in a symmetric system (with $R-L=0$ ) the eigenvalues do not have an imaginary part, and hence the uniform flow cannot undergo a Hopf bifurcation for any number of compartments. In the same context, we note that the above proof also holds for $K=2$, with (purely real) eigenvalues $\lambda_{1}=-2(R+L)$ and $\lambda_{2}=0$, but in this case the setup is symmetric with respect to the clockwise and counterclockwise direction, and thus the symmetry parameter $\delta$ is identical to 1 by definition.

\section{CONCLUSIONS}

In this paper we have studied the crossover between uniform flow and clustering in a cyclic, compartmentalized system with a bias towards one of the two flow directions. The bias is measured by the so-called symmetry parameter $\delta$, which is 1 when the material flows with equal ease in both directions, and 0 when the flow is maximally biased towards the clockwise direction. When $\delta$ is increased the transition from free flow to clustering occurs via a Hopf bifurcation (at the critical value $\delta_{\text {cr }}$ ) and an associated intermediate stage of limit cycle dynamics. The width of the interval of $\delta$ values corresponding to the limit cycle decreases sharply with growing $K$.

The reverse transition when $\delta$ is decreased takes place abruptly (and at a value of $\delta$ below $\delta_{\text {cr }}$ ), with a sudden collapse of the clustered state, which then organizes itself in a uniform flow.

One of the central points of the paper is that the value of $\delta_{\text {cr }}$ at which the Hopf bifurcation takes place is the same irrespective of the number of compartments in the system. Our mathematical proof for this (Sec. V) shows that this result does not depend on the specific form of the flux function and may thus be transferred also to related systems such as traffic flow and other multiparticle flows in which the particles interact dissipatively.

Finally, let us come back (as promised) to the special case of $K=4$ compartments. In Fig. 15 we show the bifurcation diagram for this case together with a plot of the density oscillations corresponding to the limit cycle immediately after the Hopf bifurcation. The alternating pattern typical for any even number of compartments is evident in Fig. 15(b), including the familiar blank zone between the high-level compartments and the low-level ones (just as for six compartments; cf. Fig. 14).

What makes this case unique, however, is the fact that the limit cycle has an extremely small interval of stability. Very soon after its birth at $\delta_{\text {cr }}$ its oscillating branches converge pairwise, and from that moment on they constitute a stable, nonoscillating orbit of period 2. At $\delta=0.402$, this period-2 
orbit becomes unstable via a reverse period doubling bifurcation. The four branches of the unstable clustered state (which have come into existence by means of a saddle node bifurcation at $\delta=0.232$ ) unite with the period- 2 orbit and, in doing so, render it unstable. At this point, the system has no other choice anymore but to form a cluster.

The reverse transition, as usual, takes place abruptly at the aforementioned value $\delta=0.232$. Here the cluster ceases to exist, and the material will organize itself in a uniform flow traveling around the system.

In conclusion, we see that all cases with up to five compartments are special in one way or the other. The case $K=1$ is trivial; and all other cases with an odd number of compartments $(K=3,5, \ldots)$ show the frustration mentioned in Sec. IV, namely, that the natural tendency of the system to create a limit cycle with alternating densities is thwarted. This means that only the nonfrustrated cases with an even number of compartments can be generic. Since both $K=2$ (symmetric by definition) and $K=4$ (degenerate; see above) are still special cases, this leaves $K=6$ as the first truly "generic" case.

\section{ACKNOWLEDGMENTS}

We wish to thank Dimitris Razis, John Tsamopoulos, Tassos Bountis, and Vasilis Papageorgiou for their interest in our work and stimulating discussions. We also thank Marcel Kloosterman for working together with us on the initial stages of this research, a considerable number of years ago, when we first got interested in the flow of vibrofluidized granular materials through a tilted array of connected compartments. G.K. and K.v.d.W. acknowledge partial financial support from the research project "MACOMSYS," grant code MIS 379337 , which is cofinanced by the European Union (European Social Fund) and Greek national funds through the Operational Program "Education and Lifelong Learning" of the National Strategic Reference Framework-Research Funding Program THALES: Investing in knowledge society through the European Social Fund.
[1] B. S. Kerner and P. Konhäuser, Structure and parameters of clusters in traffic flow, Phys. Rev. E 50, 54 (1994).

[2] D. Helbing, Traffic and related self-driven many-particle systems, Rev. Mod. Phys. 73, 1067 (2001).

[3] I. Goldhirsch and G. Zanetti, Clustering instability in dissipative gases, Phys. Rev. Lett. 70, 1619 (1993).

[4] J. Eggers, Sand as Maxwell's demon, Phys. Rev. Lett. 83, 5322 (1999).

[5] Y. Sugiyama, M. Fukui, M. Kikuchi, K. Hasebe, A. Nakayama, K. Nishinari, S. Tadaki, and S. Yukawa, Traffic jams without bottlnecks: Experimental evidence for the physical mechanism of the formation of a jam, New J. Phys. 10, 033001 (2008).

[6] Yu.B. Gaididei, R. Berkemer, J. G. Caputo, P. L. Christiansen, A. Kawamoto, T. Shiga, M. P. Sørensen, and J. Starke, Analytical solutions of jam pattern formation on a ring for a class of optimal velocity traffic models, New J. Phys. 11, 073012 (2009).

[7] M. R. Flynn, A. R. Kasimov, J.-C. Nave, R. R. Rosales, and B. Seibold, Self-sustained nonlinear waves in traffic flow, Phys. Rev. E 79, 056113 (2009).

[8] L. C. Davis, Jam emergence on a circular track in a car-following model, Physica A 390, 943 (2011).

[9] A. Tordeux, M. Roussignol, and S. Lassarre, Linear stability analysis of first-order delayed car-following models on a ring, Phys. Rev. E 86, 036207 (2012).

[10] M. Moussaïd, E. G. Guillot, M. Moreau, J. Fehrenbach, O. Chabiron, S. Lemercier, J. Pettré, C. Appert-Rolland, P. Degond, and G. Theraulaz, Traffic instabilities in selforganized pedestrian crowds, PLoS Comput. Biol. 8, e1002442 (2012).

[11] Y. B. Gaididei, R. Berkemer, C. Gorrida, P. L. Christiansen, A. Kawamoto, T. Shiga, M. P. Sørensen, and J. Starke, Complex spatiotemporal behavior in a chain of one-way nonlinearly coupled elements, Discrete Contin. Dyn. Syst. Ser. S 4, 1167 (2011).

[12] J. L. Laugesen and E. Mosekilde, Emergence of oscillatory dynamics, in Biosimulation in Biomedical Research, Health
Care and Drug Development, edited by E. Mosekilde, O. Sosnovtseva, and A. Rostami-Hodjegan (Springer, New York, 2012), pp. 69-95.

[13] G. Nicolis and I. Prigogine, Exploring Complexity: An Introduction (Freeman, New York, 1989).

[14] D. M. Keenan and J. D. Veldhuis, A biomathematical model of time delayed feedback in the human male hypothalamicpituitary-Leydig cell axis, Am. J. Physiol.- Endocrinology and Metabolism 275, E157 (1998).

[15] N. Rapin, E. Mosekilde, and O. Lund, Bistability in autoimmune diseases, Autoimmunity 44, 256 (2011).

[16] The cluster may be formed in any one of the $K$ compartments, with equal probability, depending on the precise position of the density enhancement at the moment when the critical level is exceeded.

[17] K. van der Weele, G. Kanellopoulos, Ch. Tsiavos, and D. van der Meer, Transient granular shock waves and upstream motion on a staircase, Phys. Rev. E 80, 011305 (2009).

[18] G. Kanellopoulos and K. van der Weele, Subcritical pattern formation in granular flow, Int. J. Bifurcation Chaos 21, 2305 (2011).

[19] G. Kanellopoulos and K. van der Weele, Critical flow and clustering in a model of granular transport: The interplay between drift and antidiffusion, Phys. Rev. E 85, 061303 (2012)

[20] K. van der Weele, W. Spit, T. Mekkes, and D. van der Meer, From granular flux model to traffic flow description, in Traffic and Granular Flow '03, edited by S. Hoogendoorn, S. Luding, P. H. L. Bovy, M. Schreckenberg, and D. E. Wolf (Springer, Berlin, 2004), pp. 569-578.

[21] D. van der Meer, Compartmentalized granular gases: Clustering and directed transport, Ph.D. thesis, University of Twente, Enschede, The Netherlands, 2004.

[22] D. van der Meer, K. van der Weele, P. Reimann, and D. Lohse, Compartmentalized granular gases: flux model results, J. Stat. Mech.: Theor. Exp. (2007) P07021. 
[23] K. van der Weele, D. van der Meer, M. Versluis, and D. Lohse, Hysteretic clustering in granular gas, Europhys. Lett. 53, 328 (2001).

[24] D. van der Meer, K. van der Weele, and D. Lohse, Bifurcation diagram for compartmentalized granular gases, Phys. Rev. E 63, 061304 (2001).

[25] D. van der Meer, K. van der Weele, and D. Lohse, Sudden collapse of a granular cluster, Phys. Rev. Lett. 88, 174302 (2002).

[26] K. van der Weele, Granular gas dynamics: How Maxwell's demon rules in a non-equilibrium system, Contemp. Phys. 49, 157 (2008).

[27] U. Marini Bettolo Marconi and A. Puglisi, Statistical mechanics of granular gases in compartmentalized systems, Phys. Rev. E 68, 031306 (2003).

[28] K. C. Chen, C. C. Li, C. H. Lin, and G. H. Guo, Clustering and phases of compartmentalized granular gases, Phys. Rev. E 79, 021307 (2009).
[29] R. Liu, Y. Li, and M. Hou, Oscillatory phenomena of compartmentalized bidisperse granular gases, Phys. Rev. E 79, 052301 (2009).

[30] Y. Li, M. Hou, and P. Evesque, Directed clustering in driven compartmentalized granular gas systems in zero gravity, J. Phys.: Conf. Ser. 327, 012034 (2011).

[31] E. Opsomer, M. Noirhomme, N. Vandewalle, and F. Ludewig, How dynamical clustering triggers Maxwell's demon in microgravity, Phys. Rev. E 88, 012202 (2013).

[32] S. H. Strogatz, Nonlinear Dynamics and Chaos (Perseus, Cambridge, 2000).

[33] R. M. Corless, G. H. Gonnet, D. E. G. Hare, D. J. Jeffrey, and D. E. Knuth, On the Lambert W function, Adv. Comput. Math. 5, 329 (2002).

[34] M. Drazin, Some generalizations of matrix commutativity, Proc. Lond. Math. Soc. 3, 222 (1951). 